\title{
Are Fresh Stillbirths from Caesarean Sections Associated with Socioeco- nomic Status? A Case-Control Study at the University Teaching Hospital in Lusaka, Zambia
}

\author{
Chanda $K^{1}, 2$, Shanzi $A^{1,2}$ and Vwalika $B^{1},{ }^{2}$
}

1. The University of Zambia, School of Medicine, Department of Obstetrics and Gynaecology, Lusaka

2. University Teaching Hospital, Department of Obstetrics and Gynaecology, Lusaka

\author{
Address of Correspondence:
}

Kenneth Chanda, 'University of Zambia, School of Medicine, Department of Obstetrics and Gynaecology, Lusaka, Zambia. Cell: +260977433942; Email: thekencha@gmail.com

\begin{abstract}
Introduction: Stillbirths occur worldwide at a rate of 3.2 million per year. There is, however, lack of interest in stillbirths, especially those from caesarean sections. The discrepancy of high stillbirth rates from caesarean sections in low to middle-income countries compared to developed countries may indicate the influence of socioeconomic status. This study was designed to investigate if the socioeconomic status is associated with caesarean section fresh stillbirths. Methods: The study involved 330 women who underwent emergency caesarean sections from October 2013 to August 2014. Of these, 110 were cases, and 220 were controls. Data collection was done using an interviewer-administered questionnaire in the immediate postoperative period.
\end{abstract}

Results: Low socioeconomic status was associated with increased odds of caesarean section fresh stillbirths. The association was, however, not statistically significant.

Keywords: Fresh, stillbirths, caesarean sections, socioeconomic status

\section{IINTRODUCTION}

The World Health Organisation (WHO) ${ }^{1}$ defines stillbirth as delivering a baby without signs of life at or after 28 weeks' gestation age or weighing 1000 grams or more. A stillbirth can either be macerated or fresh ${ }^{2}$. A macerated stillbirth is defined as one having degenerative skin changes and is presumed to have occurred 12 hours or more before delivery, whereas a fresh stillbirth is one having no such changes and is presumed to have occurred within 12 hours of delivery ${ }^{2}$.
Stillbirths are a worldwide problem with a global estimate in 2015 of about 2.6 million and a daily incidence of more than 7178 deaths, with the majority of about $98 \%$ occurring in low to middle-income countries ${ }^{1}$. It has further been noted that the most significant risk is in the intrapartum period, which accounts for about half of all stillbirths ${ }^{1}$. The dire situation in Zambia was illustrated by Turnbull et al. ${ }^{3}$, who conducted a study on the causes of stillbirths, neonatal deaths and early childhood deaths. In this study, the rate of stillbirths was 27 per 1000 deliveries.

Despite the importance of neonatal and maternal deaths rising on the worldwide agenda, stillbirths have comparatively received little attention ${ }^{4}$. This lack of interest in stillbirths is despite high stillbirth rates of 40 per 1000 total births in the Middle East and Africa ${ }^{5}$. Even though caesarean sections are expected to be associated with low rates of stillbirths, a national review of caesarean sections in Ethiopia between 2008 and 2009 showed a $14 \%$ prevalence of stillbirth and early neonatal deaths ${ }^{4,6,7}$. A similar trend of high perinatal mortality at caesarean section exists in Sub-Saharan Africa and is associated with major perioperative complications not seen in developed countries ${ }^{7}$. Stillbirths from caesarean sections at the University Teaching Hospital are not uncommon with the records showing 173 and 151 stillbirths in 2011 and 2012 respectively.

This discrepancy of high stillbirth rates from caesarean sections in low to middle-income countries as compared to developed countries raises the question of whether the socioeconomic status of the mothers plays a role. The association between stillbirths and socioeconomic status has been observed in general ${ }^{5,9}$. However, there is 
scanty data on stillbirths arising from caesarean deliveries and as to whether these are associated with socioeconomic status ${ }^{5,9}$. Furthermore, most stillbirth studies combine vaginal and caesarean deliveries births with no distinction between fresh and macerated stillbirths, and early neonatal deaths ${ }^{4}$. This study was designed to investigate if socioeconomic status of mothers in Lusaka is associated with fresh stillbirths at caesarean section.

\section{MATERIALS AND METHODS}

This was a case-control study at the University Teaching Hospital in Lusaka between October 2013 and August 2014 enrolling 330 women who delivered via emergency caesarean section. Cases were women who delivered a fresh stillbirth at a gestation age of at least 28 weeks or weighed at least 1000 grams. A receiving midwife identified a fresh stillbirth as one having no signs of life and no degenerative skin changes ${ }^{1}$. Controls were mothers who had live births from caesarean section done during the same period. The proxy measures for socioeconomic status were the area of residence, level of education and occupation.

Women who delivered stillbirths received bereavement counselling. Following counselling, the protocol and procedures of the study were explained to them. They were allowed to ask questions before being invited to take part in the study. A written consent was obtained from those who agreed to take part. Medical records and an interviewer-administered questionnaire were used to collect information from all participants. Permission was sort from UTH and University of Zambia Biomedical Research Ethics Committee [UNZABREC (Ref No. 015-07-13)].

\section{Statistical methods}

The data collected was entered into Stata for analysis. Descriptive analysis was performed on the demographic parameters, and appropriate tables were produced. Chi-squared test was performed to test if there was an association between residence, education level and occupation. Chi-square test was also performed to test the association between the measures of socioeconomic status and odds of stillbirths. Logistic regression was used to study the effect of various confounders (age, parity, foetal heat, caesarean section category and birth weight) on the association between stillbirths and socioeconomic variables.
All tests were at $95 \%$ confidence interval and a $p$-value less than 0.05 was considered statistically significant.

\section{RESULTS}

A total of 2,876 caesarean sections were performed during the study period. There were 112 fresh stillbirths who fulfilled the eligibility criteria of which two clients declined to take part, translating into a response rate of $98.21 \%$. There were two controls per case giving a total number of 330 participants (220 controls). Chi-square test indicated a strong association between education level, occupation and residence $(p>0.001)$.

Table 1 shows the demographics and baseline foetal and maternal characteristics. The mean maternal age was 29.46 years for cases and 26.91 years in the controls ( $p$ 0.001). High parity was more common among mothers with stillbirths (25.45\%) compared to live births (15.45\%) with a p-value of 0.028 . The mean gestation age for stillbirths was 29.46 weeks and that for live births was 38.22 years ( $p$ 0.002). Differences were also seen in the fetal heart on admission ( $p$-value < 0.001). There was no difference statistically in the two groups regarding marital status, miscarriages, hypertension and their reason for delivery at UTH. Table 2 illustrates the crude association and adjusted odds between measures of socioeconomic status with fresh stillbirths. The results show that there was increasing odds of stillbirths in low socioeconomic status patients compared to high socioeconomic status. Regarding residence, participants from high-density residential had one and half times more odds of fresh stillbirth compared to low-density residential areas $(\mathrm{OR}=1.22$, $\mathrm{P}=0.748$ and $\mathrm{CI}=0.36-4-14)$. Similar trends were also observed in the education status with the least educated being the most disadvantaged $(\mathrm{OR}=1.56$, $\mathrm{p}=0.345$ and $\mathrm{CI}=0.62-3.96)$. Despite this general trend, unemployment was associated with reduced odds of having a stillbirth $(\mathrm{OR}=0.91, \mathrm{p}=0.800$ and $\mathrm{CI}=0.44-1.87)$. Theses associations were however not statistically significant (OR $<2$ and $p$-value $>$ 0.001 )

\section{DISCUSSION}

Literature shows fresh stillbirth rates to be about 12 per 1000 caesarean sections ${ }^{10}$. However, our study showed a higher prevalence of about 38 fresh 
stillbirths per 1000 caesarean deliveries performed at the University Teaching Hospital. This study showed increased odds of fresh stillbirths among those women with lower social-economic status though this association was not statistically significant. This is comparable to the results in literature ${ }^{5,8}$.

The demographic profile of cases and controls showed significant variations in age and parity. In line with the findings by McClure et al, ${ }^{6}$ cases were significantly older than those in the comparison group with mean ages of 29.49 years (SD 6.51) and 26.91 years (SD 6.29) respectively. Furthermore, $25.45 \%$ of those who had fresh stillbirths were of high parity as opposed to only $15.45 \%$ of those with live births. These trends were also observed in literature ${ }^{6}$. However, the two groups were similar with regards to marital status and previous miscarriages.

With regards to foetal characteristics, differences were seen in gestation age and fetal heart rate on admission. The mean gestation age for stillbirths of 36.22 weeks was lower than that of live births of 38.22 weeks $(\mathrm{P}=0.002)$. In this line, Zhang et al. ${ }^{11}$ proposed that preterm delivery is likely to be associated with either maternal or fetal problems, and thus these babies could have been seriously compromised and therefore growthrestricted ${ }^{11}$. The majority of live births $(95.00 \%)$ had a normal fetal heart rate on admission as opposed to only $50 \%$ in the stillbirth group. The remainder of the stillbirths either had no fetal heart (28.81\%) or had fetal bradycardia $(18.80 \%)$. This finding could suggest the interplay of factors in labour with social factors in the aetiology of fresh stillbirths. The fetal and maternal characteristic differences may account for the difference in outcomes other than the socioeconomic status. These were therefore treated as confounders and controlled for in the logistic regression.

In our multivariate analysis using logistic regression, it was found that the odds of the having a fresh stillbirth increased with decreasing level of socioeconomic status. This trend was seen in education level and area of residence but not in occupation. For example, those with primary education were one and half times more likely to have a stillbirth compared to those who attained tertiary education $(\mathrm{OR}=1.56 \mathrm{p}=0.345 \mathrm{CI}=0.62$ -
3.39). Notably, women with low education were generally of higher parity which has been cited in the literature as a significant determinant for stillbirths $(\mathrm{p}<0.001)$. However, this trend was not apparent in the participants without any form of education. Therefore, it appears that the level of education could be a confounder associated with stillbirths, where high parity could be the main cause since high parity is associated with low education levels. Area of residence had similar trends to that seen in education achievement with those from high-density areas being at the most significant risk $(\mathrm{OR}=1.22, \mathrm{p}=0.748 \mathrm{CI}=0.36-4.41)$. Being unemployed showed a $10 \%$ reduction in the odds of a fresh stillbirth (OR=0.91, $\mathrm{p}=0.800$, $\mathrm{CI}=0.44-1.87)$. This was, however, not statistically significant.

Despite literature emphasising on the association between stillbirths and socioeconomic status, our results showed that the outcomes in the two groups were statistically similar ${ }^{5,8}$. This trend was seen in all the parameters used as the measure of socioeconomic status in this study.

\section{CONCLUSION}

Although this study showed that low socioeconomic status was associated with increased odds of caesarean section fresh stillbirths, this association was not statistically significant. This may suggest that fresh stillbirths at caesarean section are related to happenings during the antenatal period and circumstances surrounding labour and delivery.

\section{ACKNOWLEDGEMENTS}

We feel a deep sense of gratitude to the Medical Education Partnership Initiative (MEPI) for the financial support to wind up data collection and for data analysis. Additionally, we extend our appreciation to our research assistant Sr. Esnelly C. Ngoma, for her hard work during data collection.

\section{REFERENCES}

WHO, Maternal, Newborn, Child and Adolescent Health Data, [Internet]. [cited 2019 Dec 6]:11. Available from: https://www.who.int/ maternal_child_adolescent/epidemiology/ stillbirth/en/ 
Stringer E, Vwalika B, Killam W, Giganti M, Mbewe R, Chi B, Chintu N, Rouse D, Goldenberg R, Stringer J. Determinants of Stillbirth in Zambia, The American College of Obstetricians and Gynecologists. 2011; 117(5): 1151-1159

Turnbull E, Lembalemba M, Guffey M, BoltonMoore C, Mubiana-Mbewe M, Chintu N. Causes of stillbirth, neonatal death and early childhood death in rural Zambia by verbal autopsy assessments, Tropical Medicine and International Health. 2011; 16 (7): 894-901

Lawn J, Gravett M, Nunes T, Rubens C, Stanton C. Global report on preterm birth and stillbirth (1 of 7): definitions, description of the burden and opportunities to improve data, BMC pregnancy and child health. 2010; 10(1)

Reese A, Kutschmann M, Razum O and Spallek J. Stillbirth differences according to regions of origin: an analysis of the German perinatal database, 2004-2007, BMC Pregnancy and Childbirth. 2011; 11(63) 1471-2393

McClure E, Pasha O, Goudar S, Chomba E, Garces A, Tshefu A. Epidemiology of stillbirth in lowmiddle income countries: A Global Network Study, Acta obstetrica et Gynecology. 2011; 90: $1379-85$
Feresu A.S, Harlow S.D, Welch K and Gillespie B.W. Incidence of stillbirth and perinatal mortality and their associated factors among women delivering at Harare Maternity Hospital, Zimbabwe: a cross-section retrospective analysis, Biomed Central. 2005; 5(9)

Fenton P, Whitty C. and Reynolds F. Caesarean section in Malawi: prospective study of early maternal and perinatal mortality, British Medical Journal. 2003; 327: 537

Luque-Fernandez M, Lone N, Gutie' rrez-Garitano I, Bueno-Cavanillas A. Stillbirth risk by maternal socioeconomic status and country of origin: a population-based observational study in Spain, 2007-08, European Journal of Public Health. 2011; 22(4) 524-529

Archana S, Bukola F, M'lmunya J, Amokrane, Nafiou I. and Neves I. Cesarean delivery outcomes from the WHO global survey on maternal and perinatal health in Africa, International Journal of Gynaecology and Obstetrics. 2009; 10.1016/j.iigo.2009.08.01

Zhang X, Joseph KS, Cnattings S and Krammer M. Birth weight differences between preterm stillbirths and live births; an analysis of population-based studies from the U.S. and Sweden, Biomedical Central. 2012; 12 (119) 


\section{FIGURES AND TABLES}

Table 1. Maternal Demographics and Baseline Characteristics

\begin{tabular}{|l|c|c|c|}
\hline Variable & FSBs (110) & Live (220) & p-Value \\
\hline Mean age (Years) & $29.46(\mathrm{Sd} 6.51)$ & $26.91(\mathrm{Sd} 6.29)$ & 0.001 \\
\hline Marital status (Married) & $104(94.55 \%)$ & $202(91.82 \%)$ & 0.368 \\
\hline Parity & $82(74.55 \%)$ & $186(84.55 \%)$ & 0.028 \\
\hline Low parity & $28(25.45 \%)$ & $34(15.45 \%)$ & \\
\hline High parity & $17(15.45 \%)$ & $30(13.64 \%)$ & 0.656 \\
\hline Miscarriages (1 or more) & $11(10.00 \%)$ & $31(14.09 \%)$ & 0.119 \\
\hline Reason of UTH delivery & $97(88.73 \%)$ & $176(80.00 \%)$ & \\
\hline Booked & $02(1.82 \%)$ & $13(5.91 \%)$ & \\
\hline Referred & $20(18.18 \%)$ & $38(17.27 \%)$ & 0.619 \\
\hline Self referral & $36.22(\mathrm{sd} 4.08)$ & $38.22(\mathrm{sd} 2.86)$ & 0.002 \\
\hline Hypertensive & $31(28.81 \%)$ & $1(0.45 \%)$ & 0.001 \\
\hline Mean Gestation age (Weeks) & $20(18.80 \%)$ & $7(3.18 \%)$ & \\
\hline Admission foetal heart & $59(53.64 \%)$ & $212(91.36 \%)$ & \\
\hline Absent &
\end{tabular}

Table 2. Association of Socioeconomic Status and stillbirth

\begin{tabular}{|l|c|c|c|c|c|c|c|}
\hline & Odds & Crude OR & P-value & 95\%C. Int & *Adj. OR & p-value & C. interval \\
\hline Residence & 0.36 & & & & & & \\
\hline High & 0.35 & 0.99 & 0.984 & $0.31-3.13$ & 0.87 & 0.837 & $0.22-3.39$ \\
\hline Medium & 0.56 & 1.57 & 0.401 & $0.55-4.51$ & 1.22 & 0.748 & $0.36-4.14$ \\
\hline Low & \multicolumn{7}{|l|}{} \\
\hline Education Level \\
\hline Tertiary & 0.39 & & & & & & \\
\hline Secondary & 0.42 & 1.06 & 0.878 & $0.50-2.23$ & 1.15 & 0.761 & $0.45-2.94$ \\
\hline Primary & 0.65 & 1.65 & 0.177 & $0.79-3.42$ & 1.56 & 0.345 & $0.62-3.93$ \\
\hline None & 0.38 & 0.98 & 0.969 & 0.293 .29 & 0.39 & 0.351 & $0.05-2.82$ \\
\hline Occupation & & & & & & \\
\hline Employed & 0.55 & & & & & & \\
\hline Unemployed & 0.49 & 0.24 & 0.639 & $0.51-1.41$ & 0.91 & 0.800 & $0.44-1.87$ \\
\hline
\end{tabular}

*Logistic regression performed with adjusting for maternal age, parity, foetal heat, caesarean section category and birth weight. 